\title{
0 uso de ferramentas dinâmicas como forma de auxiliar a resolução de um problema: uma revisão teórica à luz da Teoria da Aprendizagem Significativa
}

Vinicius Machado

vinmac@utfpr.edu.br

Universidade Tecnológica Federal do

Paraná (UTFPR), Ponta Grossa, Paraná,

Brasil.

Claudio Mario Enrique

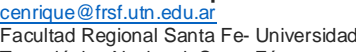
Tecnológica Nacional, Santa Fé,

Argentina.

Pedro Leineker Ochoski Machado pedmac@alunos.uttpr.edu.br Universidade Tecnológica Federal do Paraná (UTFPR), Ponta Grossa, Paraná, Brasil.

\section{RESUMO}

RESUMO

Apresenta-se nesse trabalho uma revisão teórica sobre o uso de problemas como estratégia de ensino e aprendizagem. Parte-se de um problema proposto por literatura especializada para o ensino de Física em cursos de Engenharia. Faz-se uma análise das informações contidas no enunciado do problema e propõe-se e se discute possíveis efeitos sobre a aprendizagem se o mesmo problema fosse apresentado aos alunos apoiado por um vídeo que reproduz a sua situação de forma dinâmica. Por fim, há uma análise dos possíveis efeitos da resolução desse mesmo problema se reproduzido em um laboratório didático.
\end{abstract}

PALAVRAS-CHAVE: Resolução de problemas; Aprendizagem significativa; Ensino de Física em Engenharia. 


\section{INTRODUÇÃO}

O ensino de Física nos cursos de bacharelado de Engenharia invariavelmente vem sendo desenvolvido por meio de aulas teóricas, resolução de problemas e / ou ensino no laboratório. Entre esses, entende-se a resolução de problemas um caso especial, pois apresenta características peculiares que lhe permite atuar como um elo entre o ensino teórico e o ensino no laboratório.

Por meio de um problema é possível reforçar o ensino dos conhecimentos teóricos à medida que esses se fazem necessários para o entendimento e a busca de sua solução. A situação proposta por um problema pode ser reproduzida no laboratório a fim de se verificar, por comparação, a validade dos resultados encontrados em uma resolução lápis-papel com a experimental e/ou confirmar a ocorrência de fenômenos trabalhados nas aulas teóricas, entre outras situações.

Nesse contexto, tem-se por objetivo geral, nesse trabalho, apresentar um estudo teórico e reflexões sobre o uso de ferramentas dinâmicas - recurso áudio visual e/ou atividade experimental - como forma de auxílio aos alunos no processo de resolução de um problema. Esse auxílio deve permitir ao aluno um melhor entendimento do enunciado do problema para que esse possa desempenhar melhor papel - material e mecanismo - potencializador de aprendizagem significativa.

\section{APRENDIZAGEM SIGNIFICATIVA E APRENDIZAGEM MECÂNICA POR MEIO DE PROBLEMAS}

Entende-se a resolução de problemas como uma área de interesse por docentes no que tange 0 ensino e aprendizagem visto que 0 processo desenvolvido pelo aluno na busca da resolução implica no processamento interno de informações. Nesse processamento, desenvolve-se uma atividade mental que opera por meio de símbolos ou representações da realidade, envolvendo elementos cognitivos de diferente natureza, tais como: percepção, atenção, memória, associação, raciocínio, tomada de decisões e criatividade.

Mas, o que é um problema? Entende-se um problema como uma situação com a qual uma pessoa se depara e vê a necessidade de disponibilizar certo esforço para buscar a sua solução. Esse esforço, quando o problema é utilizado para a promoção de aprendizagem, está relacionado à necessidade de utilização e reorganização de conhecimentos científicos prévios, desenvolvimento de cálculos, realização ou elaboração de procedimentos experimentais, entre outros, na busca de sua solução (MACHADO e PINHEIRO, 2010).

Assim, para que ocorra essa reorganização dos conhecimentos prévios, é necessário que se estabeleçam ou reforcem relações entre os conceitos, com isso, os conhecimentos se consolidam ou têm o enriquecimento de seus significados (AUSUBEL, NOVAK e HANESIAN, 1997; GARRET, 1988 e 1995; M ASSA, SÁNCHEZ e LLONCH, 2002, M ACHADO e PINHEIRO, 2010).

Assim, não se vê como um problema e sim como um exercício a proposição de um problema que, para a sua solução, exige do aluno apenas a aplicação imediata de conceitos, fórmulas e cálculos. Quando o ensino ocorre por meio de 
pessoa em um processo de observação, experimentação e / ou estudo sobre um fato ou uma informação ainda desconhecidos.

Ao realizar esses processos os fatos ou informações passam a ser conhecidos pela pessoa, ou seja, ficam retidos na sua memória e, se esses conhecimentos não contribuírem para a aquisição de novos conhecimentos, não resultaram de fato de um processo de aprendizagem desejável, mas, apenas a memorização de uma informação. Nesse caso, entende-se que o aluno sabe, mas não compreende. Por isso apresenta dificuldades em utilizá-los em situações diferentes. A aprendizagem decorrente da reorganização dos conhecimentos prévios e a decorrente da simples memorização são distintas e denominadas por Ausubel (2003), - autor da Teoria da Aprendizagem Significativa (TAS) - como significativa, no primeiro caso, e mecânica, no segundo.

Para Ausubel (1982 e 2003), M oreira (2000 e 2006), Buchweitz (2001), Costa e M oreira (2002), Guruceaga e González Garcia (2004) e Tavares (2004 e 2008) na aprendizagem entendida como significativa 0 indivíduo assimila um novo conhecimento por meio de relacionamentos com a estrutura dos conhecimentos já incorporados, chamados de subsunçores ou âncoras, e, por outro lado, na aprendizagem entendida como mecânica os conhecimentos são adquiridos aleatoriamente / arbitrariamente, por simples memorização. Ou seja, quando a aprendizagem é significativa o indivíduo é capaz de associar esse conhecimento com os conhecimentos já existentes produzindo dessa forma uma nova estrutura que é capaz de reconstruir-se à medida que novos conhecimentos são incorporados.

Por outro lado, quando o conhecimento é fruto de uma aprendizagem mecânica, ele se apresenta de forma isolada e independente e, como consequência, o indivíduo tende a conseguir apenas reproduzi-lo da mesma forma com que foi apropriado, apresentando dificuldades em aplicá-lo em contextos diferentes daquele em que originalmente the foi apresentado (TAVARES, 2008).

Por julgar que não há ensino sem aprendizagem, entende-se que, para que ocorra uma aprendizagem entendida como significativa, exige-se que se apliquem estratégias de ensino que sejam proporcionalmente significativas. Diante do exposto, questiona-se: que características deve ter o enunciado de um problema para que possa exercer a sua função de potencializar o ensino significativo?

A TAS condiciona a aprendizagem significativa à ocorrência de um ensino com 0 uso de materiais de ensino que apresentem mecanismos potenciais de aprendizagem significativa e que promovam 0 envolvimento acadêmico no processo de aprendizagem (AUSUBEL, 2003). Entende-se como material de ensino significativo um instrumento / estratégia de ensino, um texto a ser analisado, uma aula expositiva, um problema a ser resolvido, uma atividade experimental, entre outros, desde que esse instrumento / estratégia permita ao aluno a aquisição de novos conhecimentos a partir da base de conhecimentos por ele já adquiridos.

Entende-se como mecanismo de aprendizagem significativa aquele objeto de ensino que necessariamente tenha as características de material de ensino significativo, mas, também tenha sido elaborado com a função de promover a interação entre os conhecimentos pré-existentes e os novos conhecimentos que 
se quer ensinar, função essa entendida por Ausubel como ancoragem (AUSUBEL, 2003; TAVARES, 2004).

Na mesma linha de raciocínio de Ausubel e Tavares, Praia (2000, p.133) considera e indica algumas características necessárias a serem observadas para que os materiais de ensino sejam elaborados e possam cumprir a função de potencializar a aprendizagem significativa:

Os materiais introdutórios são importantes; Os materiais de aprendizagem devem estar bem organizados; As novas ideias e conceitos devem ser potencialmente significativos para 0 aluno; A ancoragem dos novos conceitos a estruturas cognitivas previamente existentes, nos alunos, tornará os novos conceitos recordáveis e, consequentemente, passíveis de serem utilizados em futuras aprendizagens.

Como proposta de material de ensino capaz de atuar como potencial de aprendizagem significativa, autores como Lucero et al (2006), Costa e Moreira (2002), Fávero e Sousa (2001), entre outros, sugerem a utilização de problemas. Lucero et al (2006, p. 87), por exemplo, afirmam que:

[...] La resolución de problemas es una actividad de innegable importancia para producir aprendizajes significativos, dado que ayuda a los estudiantes a reforzar y clarificar los principios que se enseñan, obligándolos a poner constantemente sus conocimientos a prueba y en práctica.

Costa e Moreira (2000), Fávero e Sousa (2001), por sua vez, lembram a importância de um problema como ferramenta de ensino à medida que para a sua solução os alunos precisem refletir e raciocinar. Como ocorre esse processo? Entende-se, em concordância com Moreira (1996) e Costa e M oreira (2002), que o aluno raciocina e reflete ao interpretar as representações externas do enunciado do problema captando-as e convertendo-as em representações internas, necessárias para a busca de uma solução.

Lucero et al (2006, p.87), ao seu modo, também afirmam que os problemas podem exercer a sua função de material de ensino significativo, se promoverem as reflexões e influenciarem no processo de aquisição de novas representações internas a partir de representações já existentes, entendidas como esquemas prévios : Cada vez que se diga que resolver problemas permite el desarrollo de procesos reflexivos, se está vinculando resolución de problemas con aprendizaje significativo, dado que toda reflexión acaecida en la mente pone em juego lainteracción entre los esquemas previos y la nueva información.

Costa e Moreira (2002, p.264) afirmam que os esquemas prévios ou representações já existentes desempenham fundamental papel, pois:

[...] a busca de solução de qualquer problema envolve uma readaptação do resíduo da experiência prévia frente às demandas da nova situação problemática a ser enfrentada. Se a estrutura cognitiva já possui as subsunções adequadas para permitir a reorganização do conhecimento, a resolução do problema terá cumprido o seu papel para a aprendizagem significativa.

Na mesma linha de raciocínio, Gaulin (2001, p.59) também ressalta a importância dos conhecimentos prévios do aluno, comparando-os a uma caixa contendo estratégias. 
Para una persona que resuelve problemas, conocer dos, tres,..., diez estrategias, es tener estratégias en una caja a su disposición. Frente a um nuevo problema puede utilizar tal estrategia, y si no funciona, probar com otra, como el carpintero. Y la persona que no conoce ningunaestrategia, tiene una cajá vacía, com lo que no es fácil que llegue a resolver el problema; por el contrario, si tiene, si conoce estrategias, si tiene métodos de resolución de problemas, conoce el modelo, conoce varias cosas, entonces sabe algo de cómo enfrentarse a los problemas y sabe que alternativas puede utilizar para resolver un problema.

Se o material de ensino e os mecanismos de ensino potencializam a aprendizagem, como promover 0 envolvimento do aluno? Entende-se que 0 envolvimento do aluno dá-se quando ele participa de forma voluntária e efetiva no processo de aquisição de um novo conhecimento e, por consequência, da reestruturação de sua base cognitiva. Nesse sentido, Praia (2000, p. 127) observa que no processo de ensino e aprendizagem a importância de que:

[...] o indivíduo manifeste uma disposição para a aprendizagem, ou seja, uma disposição para relacionar de forma não-arbitrária e substantiva, 0 novo material à sua estrutura cognitiva. Se o indivíduo tenciona simplesmente memorizar 0 material (mesmo que potencialmente significativo) de modo arbitrário e literal, o processo de aprendizagem, assim como o seu produto, serão meramente mecânicos.

Nesse contexto, entende-se que cabe ao professor montar o material e usar mecanismos capazes de despertar no aluno o interesse em trabalhar esse material, pois, segundo Ausubel (82 e 2003), ainda que o material e o mecanismo de ensino sejam elaborados objetivando potencializar a aprendizagem, não há garantias de que ocorra a aprendizagem esperada, se não houver o interesse do aluno. Assim, com as características de problema e não de exercícios, concordase com os autores citados quando afirmam que o ensino por meio de problemas pode potencializar a aprendizagem significativa.

Mas, seria possível associar ao uso de problemas mecanismos de ensino significativo? Parte-se, nesse caso, do entendimento de que um material de ensino tende a ser significativo à medida que contribui para ultrapassar a barreira limite do ensino no papel. Entende-se como ensino no papel aquele em que os conhecimentos são trabalhados em sala de aula por meio exclusivo da linguagem escrita.

Por que se vê o ensino no papel com limitações? Se Cinelli (2003, p.17) afirma que "A aprendizagem é mais eficiente quando os recursos são mais concretos [...]", assim, considera-se ser possível, nesse viés, afirmar que quanto menos abstrata ocorrer a exposição de um conteúdo ou, mais especificamente, um problema tende-se a conduzir o ensino de forma a obter melhores resultados. Isso como uma regra geral, mas, talvez de forma ainda mais acentuada no ensino de ciências, caso específico do ensino de Física no qual se estudam fenômenos naturais cujas características na maioria das vezes sofrem influência de diversos fatores, entre eles o tempo.

Com o passar do tempo a posição de um objeto e / ou a sua velocidade, a sua trajetória e até a sua massa podem sofrer alterações. Uma massa de gelo pode transformar-se em água e a água pode transformar-se em vapor, dependendo de quais fontes de calor estiverem envolvidas e os tipos de trocas de energia estas fontes provocarem. 
Com o passar do tempo um circuito elétrico pode queimar devido a um pico de tensão que eleva a corrente elétrica que 0 atravessa ou pode simplesmente parar de funcionar se nesse circuito houver um dispositivo de segurança como um disjuntor ou um fusível. Dessa forma, em boa parte das vezes em que se trabalha o ensino de Física por meio apenas da escrita entende-se não ser possível externar todos os meandros à cerca de um fenômeno.

\section{UM PROBLEMA MODELO}

A Figura 1 mostra um problema que é apresentado na forma de texto apoiado por uma gravura. Os dados do problema apresentam como informação 0 peso de dois corpos - o mais pesado será chamado de A e de B o mais leve - e a distância desses em relação ao chão.

Figura 1: um problema modelo
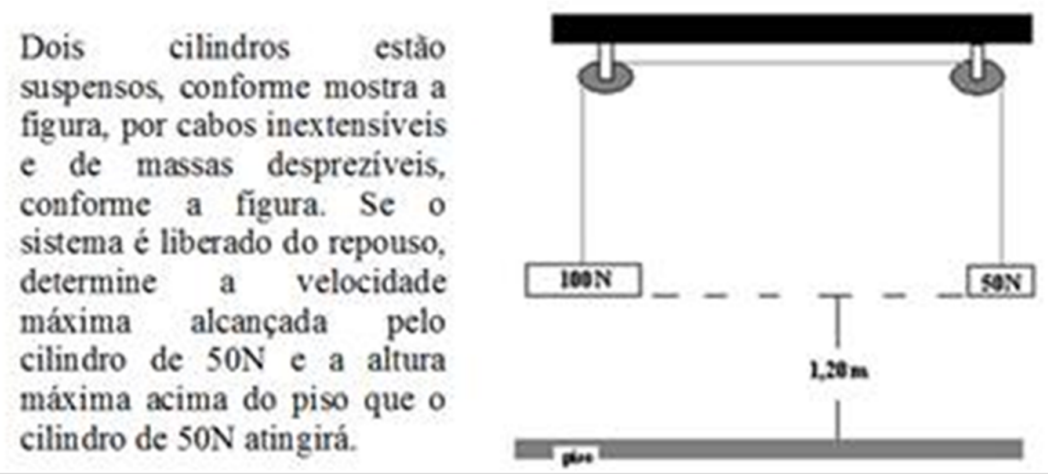

Fonte: reprodução de Beer e Johnston Jr (1980, p.123).

No enunciado não se manifesta a existência de atrito nos eixos que prendem as roldanas. Também não são informados os valores das massas das roldanas e ainda propõe que se despreze a massa do cabo em que os cilindros estão presos. Dessa forma, entende-se que o enunciado leva o aluno a considerar que o sistema do problema é conservativo em termos de energia mecânica e a desconsiderar, no balanço de energia desse sistema, a existência de energia cinética quando o sistema é liberado provocando o movimento de translação dos cilindros e de rotação das roldanas.

Quanto ao questionamento, o problema afirma, ainda que indiretamente, que ao ser liberado o sistema entra em movimento já que solicita resultados sobre velocidade e deslocamento do objeto B. Assim, na busca da resolução, o aluno passa pela necessidade de utilizar seus conhecimentos prévios para buscar a compreensão da(s) causa(s) do sistema estando em repouso, ao ser liberado, entrar em movimento: pela ação do corpo com maior intensidade de peso, poderia observar um aluno. Pelo desequilíbrio de energia potencial mecânica do sistema, poderia dizer outro.

De toda forma, entende-se que o problema, conforme exposto, supre a limitação estática que a escrita do seu enunciado e a imagem estática que Ihe apoia tem de mostrar 0 movimento. E induz / permite ao aluno perceber 0 desequilíbrio do sistema e, com esse desequilíbrio, entender o sentido do 
movimento que ocorrerá à medida que, nos seus questionamentos, pede ao aluno que determine a altura máxima atingida pelo corpo B em relação ao chão.

Assim, observa-se que a visão inicial do aluno da ocorrência de um desequilíbrio, seja pelo entendimento de que ocorrerá ação de forças diferentes no sistema ou de diferentes parcelas de energia mecânica nos corpos que constituem o sistema, permite a reorganização dos conhecimentos prévios do aluno que, adaptados ou aplicados à situação do problema, passam a criar na mente do aluno a imagem do sistema em movimento. E nessa imagem, por ele criada, compreende-se que ele vê claramente o movimento do sistema com o corpo de $50 \mathrm{~N}$ sendo puxado pelo corpo de $100 \mathrm{~N}$ até o instante em que este toca o piso.

Todavia, entende-se que o enunciado não consegue mostrar o que algumas vezes passa despercebido pelo aluno que é a ação da inércia sobre o corpo $B$, já que esse ainda apresenta um pequeno movimento mesmo depois que o corpo $\mathrm{A}$ atinge 0 piso, deixando de puxá-lo e, por isso, parte dos alunos pode responder que a maior altura atingida pelo corpo $B$ seria de apenas $2,4 \mathrm{~m}$.

A informação da ação da inércia sobre o corpo de menor peso está ausente do enunciado e muito menos pode ser observada a partir das informações dadas por uma imagem estática. Nesse caso, dariam prosseguimento à resolução do problema apenas os alunos que apoiados em seus conhecimentos prévios conseguissem visualizar o efeito da inércia sobre o corpo B. Então, como sanar essa dificuldade?

Por um lado há o entendimento de que o aluno precisa ser capaz de associar e utilizar leis, teoremas e princípios teóricos na resolução de problemas, não enxergando somente como ferramentas as equações e os cálculos. Por outro lado entende-se a necessidade de ver 0 aluno não apenas como um acadêmico de um curso de Engenharia e sim como um futuro profissional e inserido no mundo do trabalho.

Com que tipo de problemas poderá um engenheiro se defrontar? Necessariamente com aqueles apresentados por um texto apoiado por uma gravura ou com um problema real que está ali, em sua frente, ocorrendo sob o seu olhar atento? Nessa perspectiva, entende-se que uma nova ferramenta poderia ser utilizada a fim de sanar a dificuldade dos alunos que por ventura não conseguissem imaginar ou visualizar em suas mentes o efeito da inércia no movimento do sistema. Uma ferramenta que possibilitasse ao aluno literalmente ver o sistema em movimento.

\section{O USO DO VÍDEO COMO RECURSO VISUAL DINÂMICO}

Se um sistema semelhante ao proposto pela Figura 1 fosse reproduzido e tivesse o seu movimento gravado em vídeo em situação análoga à proposta pelo enunciado, criar-se-ia uma nova ferramenta de auxílio ao aluno para o entendimento e resolução do problema proposto. 0 efeito inércia sobre o bloco de menor massa seria facilmente visualizado pelo aluno. Visualizado sim, mas não ensinado / esclarecido, ou seja, o vídeo não explicaria ao aluno a razão pela continuidade do movimento do corpo B sem a simultânea ação do corpo A já que o vídeo, a princípio, apenas apresentaria uma sequência de imagens mostrando a dinâmica do movimento do sistema. 
0 vídeo por si só não ensina, é o aluno que busca, que constrói esse conhecimento à partir do uso adequado desse suporte. Tal uso supõe o recurso ao vídeo como fonte de informações, e essa exploração só acontecerá se 0 processo de inserção do vídeo tiver um sentido pedagógico para o aluno (CINELLI, 2003, p.59).

Assim, caberia ao aluno, ao observar o fenômeno por meio do vídeo, promover uma nova reorganização dos conhecimentos prévios para que, ligadas à situação observada, permitam-Ihe primeiro entender o fenômeno e, a partir desse entendimento, buscar um caminho para solucionar o problema que lhe foi proposto. Logo, entende-se que o uso do vídeo como auxílio na aprendizagem de um problema pode contribuir no processo de aprendizagem promovendo as reflexões e emprego de raciocínios. Não obstante, entende-se a necessidade de se tomar cuidado com as informações contidas no material audiovisual que se pretende utilizar. Essas informações precisam ser claras e estar apoiadas em conhecimentos já adquiridos pelos alunos. Em caso contrário, conforme afirma Rosa (2008, p.35), se pode cometer o erro de:

[...] achar que, por estarem acostumados a ver televisão, os estudantes já sejam capazes de olhar um filme de Ciências e, a partir dele, compreenderem o evento científico mostrado. É o mesmo que achar que, por alguém saber falar, seja capaz de compreender o discurso técnico.

Outro aspecto importante no uso de um vídeo é a possibilidade de interação do aluno com a ferramenta. Nesse sentido, Mandarino (2002, p.02) destaca que essa possibilidade de interação traz ao aluno "[...] a facilidade de ver, rever e analisar um produto audiovisual; a possibilidade de intervir parando, pausando, mudando o ritmo e até alterando uma sequência de imagem". Além disso, entende-se que o uso do vídeo abre outras possibilidades como, por exemplo, a de realizar leitura de medidas com maior qualidade. Ao utilizar programas que permitem a edição de vídeos pode-se reduzir a velocidade de reprodução ou até mesmo pausar e ampliar a imagem em determinado instante. Os benefícios das interações permitidas pelo uso dos vídeos como estratégia de ensino também são observados por Rosa $(2008$, p.39) no seguinte comentário:

Há certos efeitos que são melhores observados, ou somente podem ser observados, se filmados. Por exemplo, as linhas do campo magnético em um imã gigante são bem vistas quando filmadas de cima ou a vida do infinitamente pequeno só pode ser observada através de técnicas de vídeo especiais.

Para a edição de vídeos, sugere-se o uso de um software livre, ou seja, gratuito, como o VSCD Free Video Editor. O VSCD é uma ferramenta eficiente e que permite ao usuário realizar edições e também animações avançadas, porém sem requerer um grande conhecimento prévio em edições para o seu uso. Esse editor possibilita ao usuário realizar edições básicas, mas, também apresenta ferramentas de edição mais avançadas, permitindo a sobre posição de vídeos e imagens, ou seja, uma edição não linear, em que os vídeos não são exibidos conforme a ordem em que foram adicionados ao editor.

Além disso, o editor VSCD possui uma maior variedade de efeitos e uma linha de tempo que facilita o posicionamento de imagens, áudios e vídeos durante a edição. Possui maior personalização em relação a textos e também em relação ao formato final do vídeo editado. O software do editor de vídeo VSCD pode ser encontrado para download com uma simples busca na internet, por 
exemplo: http://www.videosoftdev.com/free-video-editor/download . Para o usuário pouco acostumado ao uso de recursos de edição, sugere-se visualizar tutorias disponíveis na web, realizados pelos próprios usuários que as utilizam e os disponibilizam em sites de compartilhamentos de vídeos, exemplo: https://www.youtube.com/watch?v=|Te10RjA-18 .

Assim, utilizando-se de programas de edição de vídeos entende-se que 0 docente pode construir uma nova ferramenta de apoio ao enunciado do problema, ferramenta essa bastante importante no processo de potencialização do ensino na busca de uma aprendizagem significativa.

\section{A RESOLUÇÃO DE PROBLEMAS NO LABORATÓRIO}

Parte-se do entendimento de que ao usar as atividades de ensino no laboratório como uma estratégia de ensino abre-se novos recursos. E esses recursos atuam tanto na perspectiva de permitir novos mecanismos de ancoragem quanto na possibilidade de, por meio desses mecanismos, levar o aluno a atuar de forma mais ativa e, portanto, mais envolvida nesse processo em busca da aquisição de novos conhecimentos. Mas, o ensino no laboratório por si só potencializa a aprendizagem significativa? Da Silva e Nunez (2002) e Ausubel (2003) afirmam que não. Esses autores afirmam que existem condições para que o trabalho experimental deixe de ser uma simples comprovação de conhecimentos e passe a se transformar numa atividade de ensino significativa e motivadora. Ausubel (2003, p.52) reconhece as potencialidades das atividades experimentais, contudo alerta que:

[...] 0 trabalho laboratorial e a resolução de problemas não são experiências genuinamente significativas, a não ser que se construam numa base de conceitos e de princípios claramente compreendidos na disciplina em questão e a não ser que as operações constituintes sejam, elas próprias, significativas.

Dessa forma, entende-se que Ausubel (2003) critica as atividades experimentais não significativas, sendo essas aquelas propostas aos alunos sem que para o seu desenvolvimento seja necessário o relacionamento da atividade com os conhecimentos prévios dos alunos. Em que situações isso ocorreria?

Quando a atividade experimental é passada aos alunos na forma de receita e o aluno em seu papel desempenha apenas a função de reproduzir em laboratório, sem produzir ou investigar (DA SILVA e NUNEZ, 2002). Essas são situações em que se exige do aluno apenas habilidades técnicas para a realização de medições previamente estabelecidas pelo roteiro.

0 aluno realiza as medidas de acordo e na sequência pré-estabelecida e realiza cálculos sem necessariamente a ocorrência de reflexões sobre os resultados encontrados e do(s) fenômeno(s) físico (s) envolvido(s) na atividade. Ao contrario dessa situação, Da Silva e Nunez vêm as atividades em laboratório como uma estratégia de ensino capaz de potencializar a aprendizagem significativa agindo tanto como material de ensino quanto pela sua capacidade de instigar o aluno a ter uma maior participação no processo de aquisição de conhecimentos: 
[...] a atividade experimental pode-se converter numa atividade cognoscitiva criadora e, para isso, não se devem utilizar tarefas reprodutivas, mas investigativas e produtivas, nas quais possam ser construídos e empregados os conhecimentos assimilados. Percebe-se nessa proposta o intuito de transferir ao aluno parte da responsabilidade no processo de ensino e aprendizagem promovendo, dessa forma, uma maior participação/envolvimento no processo (2002, p.1199).

Dessa forma, para que as atividades experimentais exerçam sua função estratégica de potencializar a aprendizagem significativa é fundamental que sejam elaboradas de forma a trazer questionamentos aos alunos: Como fazer isso? Para quê fazer isso? Por que fazer dessa forma e não daquela outra? E, quando forem utilizadas como auxílio ao enunciado de um problema, devem contribuir para responder questões como: Os valores estão corretos? Por que se desconsiderou o efeito de determinada grandeza? Como se obteve aquele valor tabelado? Como comprovar tal afirmação? Nesse sentido, observa-se que as experiências de laboratórios devem deixar de ser um trabalho puramente experimental e devem estar integradas com outros aspectos da atividade científica. Entre esses aspectos, destacam-se, conforme citam Carrascosa et al (2006), a necessidade de adequar o nível de dificuldade das situações problema aos estudantes, criar condições para favorecer aos alunos o entendimento da relevância da situação problema em seu estudo, potencializar não somente a análise quantitativa, mas também a qualitativa sobre os resultados encontrados, criar hipóteses a serem investigadas pelos alunos, criar condições que potencializem a dimensão coletiva do trabalho científico organizando equipes e promover a interação entre essas equipes, entre outros...

Entende-se que ao propor a resolução do problema indicado na Figura 1 no laboratório cria-se um mecanismo de ancoragem que imbrica os conhecimentos subsunçores do aluno aplicados na atividade aos novos conhecimentos que por ele serão adquiridos em função da atividade desenvolvida. Essa ancoragem torna-se necessária para que, por exemplo, o aluno possa experimentalmente encontrar / conferir / determinar os valores dos dados contidos no enunciado do problema. Usando o problema apresentado na Figura 1, 0 aluno pode, por exemplo, conferir o peso dos corpos A e B por meio de uma atividade experimental obtendo a medida direta usando um dinamômetro ou obtendo a medida indireta, medindo a massa e usando o valor da aceleração da gravidade tabelada local, aplicando esses valores à equação do peso, por eles já conhecida. Se não houver um valor pré-determinado no local para a aceleração da gravidade, 0 aluno pode utilizar recursos / instrumentos / aparelhos do laboratório para, aplicando seus subsunçores, determinar esse valor de forma prática.

Outra possibilidade aberta pela atividade no laboratório seria, ainda usando o problema proposto, a de permitir ao aluno exercer a sua criticidade sobre as informações repassadas no enunciado escrito. No laboratório o aluno pode realizar testes para verificar se o fio que une os pesos é inextensível e se amassa desse fio realmente não apresenta importância no processo a ponto de ser considerada desprezível.

Também pode fazer testes comparando o movimento do sistema quando apoiado e quando não apoiado por roldanas tendo como base para a análise dos resultados obtidos seus conhecimentos sobre atrito. Ou pode medir a massa das roldanas e verificar se em função de um valor muito pequeno a energia cinética 
no instante da rotação das roldanas efetivamente tem valor tão pequeno a ponto de ser possível desconsiderá-la. Nessa situação, o aluno precisaria levar em consideração outros conceitos como o do momento de inércia e da cinemática da rotação para conseguir obter a velocidade angular das roldanas a partir da velocidade linear do sistema decorrente do movimento dos corpos A e B.

\section{CONSIDERAÇÕES FINAIS}

Parte-se do princípio de que no processo de resolução de um problema desenvolve-se uma atividade mental que opera utilizando-se de ações como percepção, atenção, memória, associação, raciocínio, criatividade e tomada de decisões. Contudo, na busca da potencialização da aprendizagem significativa decorrente dessa atividade mental, entende-se importante destacar o papel do enunciado do problema a ser resolvido. Vê-se nos pressupostos da teoria da Aprendizagem Significativa a relevância do uso por parte do aluno de seus conhecimentos prévios para, primeiro interpretar e compreender o enunciado e, posteriormente, buscar a resolução de um problema. Para tanto, os enunciados devem trazer detalhes que mostrem ou deem ao aluno todas as informações necessárias para o seu próprio entendimento e para o entendimento da real situação em que o(s) fenômeno(s) físico(s) envolvido(s) na questão do problema acontece $(\mathrm{m})$. Caso contrário, observa-se 0 uso das ferramentas dinâmicas visuais e/ou experimentais como uma forma de auxílio ao aluno. Nesse contexto, entende-se, a partir do estudo aqui apresentado, ser válido afirmar que quando há o apoio de ferramentas dinâmicas dá-se aos alunos melhores condições de interpretação e entendimento do enunciado do problema. Dessa forma, concluise que as ferramentas dinâmicas dão ao problema uma roupa nova enquanto material e mecanismo de ensino contribuindo dessa forma para dar ao problema a condição de agir como material de ensino potencializador de aprendizagem significativa. 


\title{
The use of Dynamic Tools as a Way of Helping Solving a Problem: a Theoretical Review based on the Theory of M eaningful Learning
}

\begin{abstract}
In this paper, it is presented a theoretical review based on the Theory of Meaningful Learning about using problems as a teaching and learning strategy. One starts with a problem proposed by specialized literature for the teaching of Physics in Engineering courses. An analysis is made from the information contained in the statement of the problem, and possible effects are proposed and discussed regarding the possibility of learning if the same problem was presented to the students supported by a video that plays its situation dynamically. Finally, there is an analysis of the possible effects of the resolution of that problem if the aforementioned problem is reproduced in a teaching laboratory.
\end{abstract}

KEY WORDS: Solving Problems. Meaningful Learning. Teaching Physics in Engineering. 


\title{
El uso de herramientas dinámicas como una manera de ayudar a resolver un problema: una revisión teórica a la luz de la teoría del aprendizaje significativo
}

\begin{abstract}
RESUMEN
Se presenta en este trabajo un examen teórico a la luz de la teoría del aprendizaje significativo sobre el uso de problemas como estrategia de enseñanza y aprendizaje. Parte es un problema propuesto por la literatura especializada para la enseñanza de la Física en cursos de ingeniería. Se hace un análisis de la información contenida en la declaración del problema y propone y discute los posibles efectos sobre el aprendizaje si el mismo problema se presenta a los estudiantes el apoyo de un vídeo que se reproduce su situación de forma dinámica. Por último, se hace un análisis de los posibles efectos de la resolución de esta cuestión se reproduce en una clase de laboratorio.
\end{abstract}

PALABRAS CLAVE: Resolución de problemas; Aprendizaje significativo; Enseñanza de la física en la ingeniería. 


\section{REFERÊNCIAS}

AUSUBEL, D. P. A aprendizagem significativa: a teoria de David Ausubel. São Paulo, Moraes, 1982.

AUSUBEL, D. P. Aquisição e retenção de conhecimentos: Uma perspectiva cognitiva, Lisboa, Editora Plátano, 2003.

AUSUBEL, D., NOVAK, J. Y HANESIAN, H. Psicología Educativa: Un punto de vista cognitivo. México, Editorial Trillas, 1997.

BEER, F. P., JOHNSTON Jr, E.R. Mecânica vetorial para engenheiros. M cGraw Hill Brasil, 1980.

BUCHWEITZ, B. Aprendizagem Significativa: ideias de estudantes concluintes de curso superior. Revista Investigações em Ensino de Ciências, Rio Grande do Sul, v.6, n.2, p. 133-141, 2001.

CARRASCOSA, J.; et al. Papel de la actividad experimental en la educación científica. Caderno Brasileiro de Ensino de Física., v. 23, n. 2: p. 157-181. 2006.

CINELLI, N. P. F. A influência do vídeo no processo de aprendizagem. Dissertação de M estrado. UFSC, Florianópolis, 2003.

COSTA, S. S. C.; M OREIRA, M. A. A resolução de problemas como um tipo especial de aprendizagem significativa. Atas do III Encontro Internacional sobre Aprendizagem Significativa, Peniche, 2000, p. 243-252.

COSTA, S. S. C.; M OREIRA, M. A.O Papel da M odelagem M ental dos Enunciados na Resolução de Problemas em Física. Revista Brasileira de Ensino de Física, v. 24, no. 1, Março, 2002.

DA SILVA, S. F.; NUNEZ, I.B. O ensino por problemas e trabal ho experimental dos estudantes-reflexões teórico-metodológicas. Revista Química Nova, v. 25, n. 6/ B, p. 1197-1203, 2002.

FÁVERO, M. H.; SOUSA, C. M. S. G. A resolução de problemas em Física: revisão de pesquisa, análise e proposta metodológica. Revista Investigações em Ensino de Ciências, Porto Alegre, Brasil, v. 6, n. 2, p. 143 - 196, 2001. de ciencias. Enseñanza de las Ciencias, 6(3), 224-230, 1988. 
GARRET, R. Didáctica de las Ciencias Experimentales. Alambique, 5, 6- 15. 1995.

GAULIN, C. Tendenciasactuales de laresolución de problemas. Revista Sigma, n.19, p. 51-63, 2001.

GURUCEAGA, A.; GONZÁLES GARCIA, F. Aprendizaje Significativo Y Educación Ambiental: análisis de los resultados de una práctica fundamentada teoricamente. Revista Ensenanza de las Ciências, v.22, n.1, p.115-136, 2004.

LUCERO, I. ; et al. El Análisis Cualitativo em la Resolucion de Problemas de Fisica y su Influencia enelAprendizaje Significativo. Revista Investigações em Ensino de Ciências, Rio Grande do Sul, v.2, n1. p. 85-96, 2006.

M ACHADO, V.; PINHEIRO, N. A. M. Investigando a M etodologia dos Problemas Geradores de Discussões: aplicações na disciplina de Física no ensino de Engenharia. Revista Ciência \& Educação, vol.16, n.02, 2010, p. 525-542.

M ANDARINO, M. C. F. Organizando o trabalho com vídeo em sala de aula. M orpheus. Revista Eletrônica em Ciências Humanas. Ano 1, v. 1, 2002.

M ASSA, M., SÁNCHEZ, P. Y LLONCH, E. Convergencias y divergencias en la resolución de problemas de Física: un estudio comparativo en grupos expertos, Memorias (CD) del III Taller Internacional de Didáctica sobre la Física Universitaria. DIDACFISU 2002, Cuba. 2002.

M OREIRA, M . A. M odelos mentais. Revista Investigações em Ensino de Ciências, Rio Grande do Sul, v.1, n.3, 1996, p.193-232.

M OREIRA, M . A. Aprendizagem significativa crítica. Atas do III Encontro Internacional sobre Aprendizagem Significativa, Peniche, Lisboa, p. 33-45, com 0 título original de Aprendizagem significativa subversiva, setembro de 2000.

M OREIRA, M . A. Aprendizagem significativa: da visão clássica à visão crítica. Conferência de encerramento do V Encontro Internacional sobre Aprendizagem Significativa, M adrid, Espanha, setembro de 2006.

PRAIA, J. F. Aprendizagem significativa em D. Ausubel: Contributos para uma adequada visão da sua teoria e incidências no ensino. Teoria da aprendizagem significativa. Peniche, Portugal, p. 121-134, 2000. 
SÁNCHEZ, P. El proceso de modelado en la resolución de un problema: un estudio de caso. M emorias de la XIV REF XIV. Bariloche, Argentina. 2005.

SÁNCHEZ, P. Las Representaciones Mentales en la Resolución de Problemas de Mecánica Clásica.Tesis de Doctorado. Facultad de Psicología. Madrid: UNED. 2011

TAVARES, R. Aprendizagem significativa e o ensino de ciências. Revista Ciências \& Cognição, v. 13, n.1, p. 94-100, 2008.

TAVARES, R. Aprendizagem significativa. Revista Conceitos, n. 10, p. 55-60, João Pessoa, 2004.

Recebido: 15 de julho de 2016

Aprovado: 20 de outubro de 2016.

Como citar:

MACHADO, V.; ENRIQUE, C.M.: MACHADO, P.L.O. O uso de ferramentas dinâmicas como forma de auxiliar

a resolução de um problema: uma revisão teórica à luz da Teoria da Aprendizagem Significativa. Revista

Brasileira de Física Tecnológica Aplicada, Ponta Grossa, v. 3, n.2, p. 19-34, nov./dez. 2016.

Contato:

Vinicius Machado

vinmac@utfpr.edu.br

Direito autoral: Este artigo está licenciado sob os termos da Licença Creative Commons-Atribuição 4.0

Internacional.

(c) (i) 